\title{
Decoherence in quantum systems in a static gravitational field
}

\author{
Ahmad Shariati*1, Mohammad Khorrami*2, Farhang Loran腅 \\ * Department of Physics, Alzahra University, Tehran 1993891167, Iran \\ $\dagger$ Department of Physics, Isfahan University of Technology, Isfahan 84156-83111, Iran
}

\begin{abstract}
A small quantum system is studied which is a superposition of states localized in different positions in a static gravitational field. The time evolution of the correlation between different positions is investigated, and it is seen that there are two time scales for such an evolution (decoherence). Both time scales are inversely proportional to the red shift difference between the two points. These time scales correspond to decoherences which are linear and quadratic, respectively, in time.
\end{abstract}

\section{Introduction}

Interaction of a system with its environments could result in the so called quantum decoherence, a decrease in the correlation between different parts of the system. Such interactions, could also result in increasing the correlation.

An example is the effect of a gravitational field on such correlations. Gravitational time dilations, which depend on the position, cause the phase change in different parts of the system located at different positions to be different. That could result in a decoherence, or in certain cases a coherence, that is an increase in the correlation between different parts.

Recently, this phenomena has been studied in [1] explicitly for a system of $N$ harmonic oscillators in thermal equilibrium. An interested reader may consult [1] and 2] for a comprehensive list of references, and additional arguments in favor of this observation.

Of course time dilation is not the only source of decoherence. Interaction with environment is another source. These decoherence due to scattering, occur at rates which are proportional to the square of the distance. Some typical rates have been given in [3] and [4, for example.

A quantum system in a gravitational field could affect the coherence properties of the gravitational field as well. An example is discussed in chapter 4 of [5].

Here a general quantum system is investigated, which is in a static gravitational field, and is delocalized. The system is assumed to be a small system

\footnotetext{
${ }^{1}$ e-mail: shariati@mailaps.org

2e-mail: mamwad@mailaps.org

${ }^{3}$ e-mail: loran@cc.iut.ac.ir
} 
(of a small size $\ell$ ) with internal degrees of freedom, say a molecule. By delocalization, it is meant that the state of the system is a superposition of states at different points of the space, distributed in a region of size $\Delta x$. The length scale corresponding to the gravitational field (the curvature length) is $L$. It is assumed that

$$
\ell \ll L, \Delta x
$$

$\Delta x$ could be smaller or larger than $L$, but more explicit calculations are performed for the case

$$
\Delta x \ll L
$$

It is seen that if the system is delocalized, the correlation between different parts of it located in different positions varies with time. Two time scales are obtained for these changes. Both are inversely proportional to the gravitational potential difference (or the red shift difference) between the two points. The first time scales corresponds to a change of the correlation which is linear in time, and could be either an increase or a decrease. This time scale is finite only if there are non real correlations between different points. The second time scale corresponds to a decrease in the correlation, which is quadratic in time. This time scale is inversely proportional to the energy fluctuations of the system. This is similar to the one obtained in 1 .

The scheme of the paper is the following. A system is studied which has internal degrees of freedom and is delocalized in a static gravitational field. In section 2 the Hamiltonian, and in section 3 the time evolution of such a system are investigated. In section 4 the results are specialized to the case the system is in thermal equilibrium. Section 5 is devoted to the concluding remarks.

\section{The Hamiltonian corresponding to internal de- grees of freedom}

The line element of a static gravitational field can be written as

$$
\mathrm{d} s^{2}=-c^{2} f^{2} \mathrm{~d} t^{2}+g_{i j} \mathrm{~d} x^{i} \mathrm{~d} x^{j}
$$

where $f$ and $g_{i j}$ 's depend on only $x^{i}$ 's (the spatial coordinates). An observer at rest at the point $x$ would measure the proper time $\tau$ which is related to $t$ as

$$
\mathrm{d} \tau=[f(x)] \mathrm{d} t
$$

A localized system at rest at the point $x$ could have energy levels $E_{n}$, according to the observer at the same point $x$. The Hamiltonian corresponding to these energy levels is the generator of the evolution of the proper time according to that observer. Let us denote that Hamiltonian with $H$. For a system at rest at 
the point $x$, the evolution operator would be

$$
\begin{aligned}
U & =\exp \left(\frac{\tau H}{\mathrm{i} \hbar}\right), \\
& =\exp \left\{\frac{t[f(x)] H}{\mathrm{i} \hbar}\right\} .
\end{aligned}
$$

This suggests a global Hamiltonian $\boldsymbol{H}$, which is the generator of the evolution of the time coordinate:

$$
\boldsymbol{H}=[f(X)] H,
$$

where $X$ is the position operator:

$$
\boldsymbol{H}|x, n\rangle=[f(x)] E_{n}|x, n\rangle,
$$

where $n$ labels the internal degrees of freedom.

A slightly more general version of (6) is

$$
\tilde{\boldsymbol{H}}=[f(X)] H+V(X) .
$$

That change could be interpreted as changing the reference of energy of the internal degrees of freedom by a position-dependent amount, or equivalently adding a potential energy (corresponding to a force). It will be seen, however, that this change does not affect the decoherence.

\section{The evolution}

Consider a density matrix

$$
\rho(t)=\sum_{n, n^{\prime}} \int \mathrm{d} x \mathrm{~d} x^{\prime} \rho\left(x, x^{\prime} ; n, n^{\prime} ; t\right)|x, n\rangle\left\langle x^{\prime}, n^{\prime}\right| .
$$

Evolving this density matrix with a global Hamiltonian results in

$$
\begin{aligned}
\rho\left(x, x^{\prime}, n, n^{\prime} ; t\right)= & \rho\left(x, x^{\prime}, n, n^{\prime} ; 0\right) \\
& \times \exp \left\{\frac{\left[E_{n} f(x)+V(x)-E_{n^{\prime}} f\left(x^{\prime}\right)-V\left(x^{\prime}\right)\right] t}{\mathrm{i} \hbar}\right\} .
\end{aligned}
$$

Tracing this over the internal degrees of freedom,

$$
\tilde{A}\left(x, x^{\prime} ; t\right)=\sum_{n} \rho\left(x, x^{\prime}, n, n ; t\right),
$$

it is seen that

$$
\tilde{A}\left(x, x^{\prime} ; t\right)=\exp \left\{\frac{\left[V(x)-V\left(x^{\prime}\right)\right] t}{\mathrm{i} \hbar}\right\} A\left(x, x^{\prime} ; t\right),
$$


where

$$
A\left(x, x^{\prime} ; t\right)=\sum_{n} \rho\left(x, x^{\prime}, n, n ; 0\right) \exp \left\{\frac{\left[f(x)-f\left(x^{\prime}\right)\right] E_{n} t}{\mathrm{i} \hbar}\right\} .
$$

A measure of the decoherence of the system is a decrease of $|\tilde{A}|$ in time. It is seen that

$$
\left|\tilde{A}\left(x, x^{\prime} ; t\right)\right|=\left|A\left(x, x^{\prime} ; t\right)\right| .
$$

Hence $V(X)$ does not affect the decoherence. Defining $\gamma$ and $a_{n}$ 's and $\chi_{n}$ 's through

$$
\begin{aligned}
\gamma\left(x, x^{\prime} ; t\right) & =\frac{A\left(x, x^{\prime} ; t\right)}{A\left(x, x^{\prime} ; 0\right)}, \\
\rho\left(x, x^{\prime}, n, n ; t\right) & =\tilde{A}\left(x, x^{\prime} ; t\right) a_{n}\left(x, x^{\prime} ; t\right) \exp \left[\mathrm{i} \chi_{n}\left(x, x^{\prime} ; t\right)\right],
\end{aligned}
$$

where $a_{n}$ 's are real and nonnegative and $\chi_{n}$ 's are real, it is seen that

$$
\begin{aligned}
1 & =\sum_{n} a_{n}\left(x, x^{\prime} ; t\right) \exp \left[\mathrm{i} \chi_{n}\left(x, x^{\prime} ; t\right)\right], \\
\gamma\left(x, x^{\prime} ; t\right) & =\sum_{n} a_{n}\left(x, x^{\prime} ; 0\right) \exp \left[\mathrm{i} \phi_{n}\left(x, x^{\prime} ; t\right)\right],
\end{aligned}
$$

where

$$
\phi_{n}\left(x, x^{\prime} ; t\right)=\chi_{n}\left(x, x^{\prime} ; 0\right)-\frac{\left[f(x)-f\left(x^{\prime}\right)\right] E_{n} t}{\hbar} .
$$

If $E_{n}$ 's are commensurate, then $\gamma\left(x, x^{\prime} ; t\right)$ would be periodic in time, with the period $t_{0}$ satisfying

$$
t_{0}=\frac{h}{\left[f(x)-f\left(x^{\prime}\right)\right] \varepsilon},
$$

where $\varepsilon$ is the greatest common divisor of $E_{n}$ 's.

Up to second order in time,

$$
\begin{aligned}
\gamma\left(x, x^{\prime} ; t\right)= & +\frac{\left[f(x)-f\left(x^{\prime}\right)\right] t}{\mathrm{i} \hbar} \sum_{n} E_{n} a_{n}\left(x, x^{\prime} ; 0\right) \exp \left[\mathrm{i} \chi_{n}\left(x, x^{\prime} ; 0\right)\right] \\
& +\frac{1}{2}\left\{\frac{\left[f(x)-f\left(x^{\prime}\right)\right] t}{\mathrm{i} \hbar}\right\}^{2} \sum_{n}\left(E_{n}\right)^{2} a_{n}\left(x, x^{\prime} ; 0\right) \exp \left[\mathrm{i} \chi_{n}\left(x, x^{\prime} ; 0\right)\right] \\
& +\cdots
\end{aligned}
$$


So,

$$
\begin{aligned}
\left|\gamma\left(x, x^{\prime} ; t\right)\right|^{2}= & 1+\frac{2\left[f(x)-f\left(x^{\prime}\right)\right] t}{\hbar} \sum_{n} E_{n} a_{n}\left(x, x^{\prime} ; 0\right) \sin \left[\chi_{n}\left(x, x^{\prime} ; 0\right)\right] \\
& +\left\{\frac{\left[f(x)-f\left(x^{\prime}\right)\right] t}{\hbar}\right\}^{2}\left|\sum_{n} E_{n} a_{n}\left(x, x^{\prime} ; 0\right) \exp \left[\mathrm{i} \chi_{n}\left(x, x^{\prime} ; 0\right)\right]\right|^{2} \\
& -\left\{\frac{\left[f(x)-f\left(x^{\prime}\right)\right] t}{\hbar}\right\}^{2} \sum_{n}\left(E_{n}\right)^{2} a_{n}\left(x, x^{\prime} ; 0\right) \cos \left[\chi_{n}\left(x, x^{\prime} ; 0\right)\right] \\
& +\cdots
\end{aligned}
$$

Both $\rho\left(x, x^{\prime}, n, n: 0\right)$ and $A\left(x, x^{\prime} ; 0\right)$ are real and nonnegative for $x^{\prime}=x$. Hence $\chi_{n}$ vanishes for $x^{\prime}=x$, so that up to second order in $\left(x^{\prime}-x\right)$ one has

$$
\begin{aligned}
\left|\gamma\left(x, x^{\prime} ; t\right)\right|^{2}= & 1+\frac{2\left[f(x)-f\left(x^{\prime}\right)\right] t}{\hbar}\left(x^{\prime}-x\right)\langle E \mathrm{D} \chi\rangle(x ; 0) \\
& -\left\{\frac{\left[f(x)-f\left(x^{\prime}\right)\right] t}{\hbar}\right\}^{2}(\Delta E)^{2}(x ; 0)+\cdots, \\
= & 1-\left(x^{\prime}-x\right)^{2}\left(2 \frac{[(\mathrm{D} f)(x)] t}{\hbar}\langle E \mathrm{D} \chi\rangle(x ; 0)\right. \\
& \left.+\left\{\frac{[(\mathrm{D} f)(x)] t}{\hbar}\right\}^{2}(\Delta E)^{2}(x ; 0)\right)+\cdots
\end{aligned}
$$

where D stands for differentiation and

$$
\begin{aligned}
\langle E \mathrm{D} \chi\rangle(x ; 0) & =\sum_{n} E_{n} a_{n}(x, x ; 0) \mathrm{D}_{2} \chi_{n}(x, x ; 0) . \\
(\Delta E)(x ; 0) & =\left\{\sum_{n}\left(E_{n}\right)^{2} a_{n}(x, x ; 0)-\left[\sum_{n} E_{n} a_{n}(x, x ; 0)\right]^{2}\right\}^{1 / 2} .
\end{aligned}
$$

$\mathrm{D}_{i}$ is differentiation with respect to the $i$ 'th variable. Of course $\left[a_{n}(x, x ; 0)\right]$ 's are real nonnegative and add up to unity.

The above allows for a change in coherence which is first order in time, unless $\chi_{n}$ 's vanish. But if there is no first-order change, then the coherence decreases initially. There are two time scales corresponding to the change in $\left|\gamma\left(x, x^{\prime} ; t\right)\right|$ :

$$
\begin{aligned}
t_{1} & =\frac{h}{\left|\left(x^{\prime}-x\right)^{2}[(\mathrm{D} f)(x)][\langle E \mathrm{D} \chi\rangle(x ; 0)]\right|} . \\
t_{2} & =\frac{h}{\left|\left(x^{\prime}-x\right)[(\mathrm{D} f)(x)]\right|[(\Delta E)(x ; 0)]} .
\end{aligned}
$$


In terms of proper times, these become

$$
\begin{aligned}
\tau_{1} & =\frac{h f(x)}{\left|\left(x^{\prime}-x\right)^{2}[(\mathrm{D} f)(x)][\langle E \mathrm{D} \chi\rangle(x ; 0)]\right|} . \\
\tau_{2} & =\frac{h f(x)}{\left|\left(x^{\prime}-x\right)[(\mathrm{D} f)(x)]\right|[(\Delta E)(x ; 0)]} .
\end{aligned}
$$

Both time scales are inversely proportional to $(\mathrm{D} f)(x)$.

The origin of the first order change in coherence, could be traced back to (19). It is seen that if $\chi_{n}$ 's are initially zero, then $\phi_{n}$ 's are initially zero and the coherence is maximum (it decreases with time). If $\chi_{n}$ 's are nonzero but in the opposite phase with the energy times the difference of the red shifts, then $\phi_{n}$ 's decrease with time, making the system more like the case of vanishing $\chi_{n}$ 's (vanishing $\phi_{n}$ 's), hence increasing the coherence. It could also be noted that $\phi_{n}$ 's, even if initially are real don't remain so. So, if one begins with a system with vanishing $\chi_{n}$ 's (vanishing $\phi_{n}$ 's), time evolution produces (for small times) $\phi_{n}$ 's which are in phase with the energy times the difference of the red shifts, hence decreasing the correlation further.

\section{Thermal equilibrium}

For a system localized at the point $x$, and having a local temperature $T(x)$, the density matrix is

$$
\rho=|x\rangle\langle x| \rho_{\text {in }}[T(x)]
$$

where

$$
\begin{aligned}
& \rho_{\text {in }}(T)=\frac{1}{Z_{\text {in }}(T)} \sum_{n}|n\rangle\langle n| \exp \left(-\frac{E_{n}}{k_{\mathrm{B}} T}\right), \\
& Z_{\text {in }}(T)=\sum_{n} \exp \left(-\frac{E_{n}}{k_{\mathrm{B}} T}\right) .
\end{aligned}
$$

If the system is delocalized, it is not the local Hamiltonian which is conserved. Consider changes $\Delta_{1}$ and $\Delta_{2}$ in the local energies at the points $x_{1}$ and $x_{2}$, respectively. One has

$$
\Delta_{2}=-\frac{f\left(x_{1}\right)}{f\left(x_{2}\right)} \Delta_{1}
$$

$\Delta S$, the change of entropy, would then be

$$
\Delta S=\frac{\Delta_{1}}{T_{1}}+\frac{\Delta_{2}}{T_{2}} .
$$


A necessary condition for thermal equilibrium is that $\Delta S$ be zero. This results in

$$
\left[f\left(x_{2}\right)\right] T_{2}=\left[f\left(x_{1}\right)\right] T_{1} .
$$

So a necessary condition for the thermal equilibrium would be the existence of a global (position-independent) temperature $\boldsymbol{T}$ such that the local temperatures satisfy

$$
T(x)=\frac{\boldsymbol{T}}{f(x)} .
$$

A density matrix corresponding to local temperatures $T(x)$ at each point $x$, is not unique. It should satisfy

$$
\rho\left(x, x ; n, n^{\prime}\right)=A(x, x)\left\langle n\left|\rho_{\text {in }}[T(x)]\right| n^{\prime}\right\rangle .
$$

But this does not say anything about $\rho\left(x, x^{\prime} ; n, n^{\prime}\right)$ with $x^{\prime} \neq x$. A simple choice is

$$
\rho\left(x, x^{\prime} ; n, n^{\prime}\right)=\frac{A\left(x, x^{\prime}\right)}{Z_{\mathrm{in}}\left(\boldsymbol{T} ; x, x^{\prime}\right)} \exp \left\{-\frac{E_{n}\left[f(x)+f\left(x^{\prime}\right)\right]}{2 k_{\mathrm{B}} \boldsymbol{T}}\right\} \delta_{n n^{\prime}},
$$

where

$$
Z_{\text {in }}\left(\boldsymbol{T} ; x, x^{\prime}\right)=\left\{Z_{\text {in }}\left[\frac{\boldsymbol{T}}{f(x)}\right] Z_{\text {in }}\left[\frac{\boldsymbol{T}}{f\left(x^{\prime}\right)}\right]\right\}^{1 / 2} .
$$

Such a choice results in

$$
\begin{aligned}
& \chi_{n}\left(x, x^{\prime}\right)=0 . \\
& a_{n}\left(x, x^{\prime}\right)=\frac{1}{Z_{\text {in }}\left(\boldsymbol{T} ; x, x^{\prime}\right)} \exp \left\{-\frac{E_{n}\left[f(x)+f\left(x^{\prime}\right)\right]}{2 k_{\mathrm{B}} \boldsymbol{T}}\right\} .
\end{aligned}
$$

The choice (39) for the density matrix at $t=0$, gives

$$
\gamma\left(x, x^{\prime} ; t\right)=\frac{Z_{\text {in }}(\mathcal{T})}{Z_{\text {in }}\left(\boldsymbol{T} ; x, x^{\prime}\right)},
$$

where

$$
\frac{1}{\mathcal{T}}=\frac{f(x)+f\left(x^{\prime}\right)}{2 \boldsymbol{T}}-\frac{k_{\mathrm{B}}\left[f(x)-f\left(x^{\prime}\right)\right] t}{\mathrm{i} \hbar} .
$$

One also notices that in this case,

$$
\begin{aligned}
\langle E \mathrm{D} \chi\rangle(x ; 0) & =0, \\
(\Delta E)^{2}(x ; 0) & =k_{\mathrm{B}}\left[\frac{\boldsymbol{T}}{f(x)}\right]^{2} C(x),
\end{aligned}
$$


where $C(x)$ is the local heat capacity at the point $x$, corresponding to the internal degrees of freedom. For small times, the coherence deceases quadratically in time, with a time scale

$$
\begin{aligned}
\tau_{2} & =\frac{h[f(x)]^{2}}{\left|\left(x^{\prime}-x\right)[(\mathrm{D} f)(x)]\right| \sqrt{k_{\mathrm{B}} C(x)} \boldsymbol{T}}, \\
& =\frac{h f(x)}{\left|\left(x^{\prime}-x\right)[(\mathrm{D} f)(x)]\right| \sqrt{k_{\mathrm{B}} C(x)} T(x)} .
\end{aligned}
$$

For a system not in extreme conditions (not in strong gravitational fields), one could approximate the above:

$$
\tau_{2} \approx \frac{h}{k_{\mathrm{B}} T} \sqrt{\frac{k_{\mathrm{B}}}{C}} \frac{c^{2}}{g \Delta x},
$$

where $f$ is taken to be nearly one, so that the $x$-dependences are neglected except for the derivative of $f$. $g$ is the gravitational acceleration, and use has been made of the fact that $c^{2}(f-1)$ is the gravitational potential [6], for example. If the effective number of internal degrees of freedom (those which have been excited) is neither small nor large, say considering a simple molecule in room temperature, then $C$ is of the order of $k_{\mathrm{B}}$. Then at room tempertaure,

$$
\tau_{2} \sim \frac{10^{4} \mathrm{~m}^{2} \mathrm{~s}^{-1}}{g \Delta x} .
$$

which for a system of size around $1 \mathrm{~m}$ on earth results in

$$
\tau_{2} \sim 10^{3} \mathrm{~s} .
$$

\section{Concluding remarks}

The effect of gravitational time dilation of the correlation between parts of a small quantum system located at different points was studied. It was shown that

- External potentials do not affect the correlation.

- There are in general two time scales for the change in correlation. The first time scale is finite, only if there are non-real correlations between different points. This time scale results in a change in the correlation which is first order in time, and could lead to an increase or a decrease in the correlation. The second time scale is always there, and results in a change in the correlation which is second order in time, and always leads to a decrease in the correlation.

Acknowledgement: The work of AS and MK was supported by the research council of the Alzahra University. 


\section{References}

[1] I. Pikovski, M. Zych, F. Costa, \& C. Brukner, Nature Physics 11 (2015) 668.

[2] I. Pikovski, M. Zych, F. Costa, \& C. Brukner, arXiv:1508.03296 [quant-ph] (2015).

[3] E. Joos, \& H. D. Zeh, Z. Phys. B59 (1985) 223.

[4] M. Tegmark, Found. Phys. Lett. 6 (1993) 571.

[5] D. Giulini, E. Joos, C. Kiefer, J. Kupsch, I.-O. Stamatescu, \& H. D. Zeh, "Decoherence and the Appearance of a Classical World in Quantum Theory", (Springer Verlag, 1996).

[6] C. M. Will, "Theory and Experiment in Gravitational Physics", (Cambridge University Press, 1993). 